Declaring Conflict of Interest - Current State of Affairs in the Ophthalmic Literature Peer-reviewed author version

Schaefer, Jamie Lea; AUBERT BONN, Noemie \& Craenen, Geert (2017) Declaring Conflict of Interest - Current State of Affairs in the Ophthalmic Literature. In: ACCOUNTABILITY IN RESEARCH-POLICIES AND QUALITY ASSURANCE, 24(7), p. 375-383.

DOI: $10.1080 / 08989621.2017 .1357474$

Handle: http://hdl.handle.net/1942/27528 
Accountability in Research

Policies and Quality Assurance

ISSN: 0898-9621 (Print) 1545-5815 (Online) Journal homepage: http://www.tandfonline.com/loi/gacr20

\title{
Declaring conflict of interest - current state of affairs in the ophthalmic literature
}

\author{
Jamie Lea Schaefer, Noemie Aubert Bonn \& Geert Craenen
}

To cite this article: Jamie Lea Schaefer, Noemie Aubert Bonn \& Geert Craenen (2017): Declaring conflict of interest - current state of affairs in the ophthalmic literature, Accountability in Research, DOI: $10.1080 / 08989621.2017 .1357474$

To link to this article: http://dx.doi.org/10.1080/08989621.2017.1357474

Accepted author version posted online: 26 Jul 2017.

Submit your article to this journal ๘

III Article views: 1

Q View related articles $₫$

View Crossmark data \lceil 


\section{Declaring Conflict of Interest - Current State of Affairs in the}

\section{Ophthalmic Literature.}

Jamie Lea Schaefer MD ${ }^{1}$, Noemie Aubert Bonn $\mathrm{MSc}^{2}$, Geert Craenen MD MBE ${ }^{1,3,4}$

University at Buffalo Ross Eye Institute'; Hasselt University, Faculty of Medicine and Life

Sciences $^{2}$; University at Buffalo Center for Clinical Ethics, Western New York VAMC ${ }^{4}$.

\section{CONTACT}

Geert Craenen MD MBE

Dept. of Ophthalmology; Room 627 D

WNYVAMC

3495 Bailey Avenue

Buffalo NY 14215

Email: Geert.Craenen@,VA.Gov

\section{Abstract:}

Context: The importance of transparency with financial ties in biomedical research is widely recognized, and most peer-reviewed journals require declarations of Conflicts of Interest (COI). Nonetheless, variability in the consistency of declarations of COI has been sparsely assessed.

Objectives: To assess consistency and rates of COI declarations in the ophthalmic literature and the effectiveness of journal COI policies.

Methods: We analyzed consistency and completeness of declaration of COI in the ophthalmic literature and compared the levels of completeness to specific journal requirements.

Results: 642 peer reviewed articles satisfied the inclusion criteria. In 64\%, COIs were unreported, in $25 \%$ declaration of COI was incomplete, and $11 \%$ of the articles reviewed had complete declaration of COI. Of the 33 journals in which the most frequently published authors' 
articles appeared, 10 required the authors to complete the International Committee of Medical Journal Editors (ICMJE) form or an equivalent form but this did not affect the rates of COI declaration.

Conclusions: In a random sampling of the most frequently published authors in the field of ophthalmology, declaration of COI was low and highly inconsistent. Requiring a standardized $\mathrm{COI}$ form has no significant effect on the rate of accurate COI reporting. Our findings lend support to the growing body of literature that shows that journals and editors may need to take a more active role in ensuring accurate and consistent $\mathrm{COI}$ reporting.

\section{Introduction:}

The November and December 2014 issues of Ophthalmology and the American Journal of Ophthalmology feature a joint editorial addressing ongoing issues with declaration of authors' conflicts of interest and recent changes in the recommendations of the International Committee of Medical Journal Editors (ICMJE) on financial disclosure ${ }^{1,2}$. In the editorial Liesegang and Bartley, the respective editors, commit to maintaining physician and public trust through enhanced transparency. Amongst other things, both editors argue that disclosure of interests should include all ties to industry encompassing the 36 months preceding submission, even if not specific to the submitted work. The two editors also submit that terms such as "potential conflict of interest" and "relevant financial information" lead to confusion and form an impediment to transparency.

Liesegang and Bartley advocate for universal adoption of the ICMJE form to effect this transparency. To the ICMJE, improved transparency takes the form of requiring all authors to 
disclose all financial ties and interests, and letting the readers decide on their significance for themselves. "Confusion and subjectivity in reporting financial conflict" the editors argue, now including continuing medical education presentations in their scope of comments, "would be lessened simply by disclosing everything and having the same disclosures at each presentation and each manuscript submission."1

We applaud these editors of two of the leading journals in ophthalmology for taking this strong public stance on an issue vital to the future of scientific reporting, and this leads us to wonder: How well does the literature in our own field of ophthalmology live up to these high standards?

The same December issue of Ophthalmology, for example, contains back-to-back articles in which the same author, writing on the same subspecialty topic, using the same methods and materials, reports very different financial interests. Illustrative of the findings reported in our paper, such discrepancies demonstrate the need for a better understanding of the declaration of conflict of interests (DOI) in practice.

DOI presumes an understanding of which interests may be considered conflicting, biased, or requiring special mention. Agreement on what should be declared, even amongst the publishing arm of the research enterprise, is far from universal, and DOI requirements differ dramatically from journal to journal. ${ }^{3,4}$ In the past few years, increasing efforts have been made to arrive at a consistent, common DOI format. The International committee for Medical Journal Editors (ICMJE), for example, probably provides the most highly endorsed DOI form - the ICMJE Form for Disclosure of Potential Conflicts of Interests - intended to be completed by all authors at the time of submission and to be confirmed before publication. This form ensures that each author declares any payment or services received for any aspect of the work submitted, but also any 
financial activities outside the submitted work "that could be perceived to influence, or that give the appearance of potentially influencing, what [is written] in the submitted work" ${ }^{5}$, as well as any other relationship that authors might deem relevant to declare in a format that consists of closed ended questions. ${ }^{6}$ Despite these efforts to thoroughly cover conflicts of interest, the ICMJE form and most independent journal requirements rely heavily on the authors' interpretation and judgment of what constitutes a conflicting interest, highlighting the concerns raised by Liesegang and Bartley about terms such as potential or relevant conflicts of interests.

To assess problems of consistency that may result from ambiguous demands or differing interpretations of conflict of interests we investigated the consistency in DOI for the most frequently published authors in 3 subspecialties within ophthalmology (i.e., glaucoma, macular disease, and corneal disease). To complement those results, we also analyzed journals' DOI requirements to understand the level of discrepancy that exists between the many journals used to report research in the field of Ophthalmology, and the influence of journals' requirement on DOI completeness.

For the purpose of this paper, we will define COI as "a situation in which there is a risk of bias and resulting harm, not a situation in which bias or harm necessarily occurs".?

\section{Methods:}

Using Web of Science, which features a handy publication count by author, we identified the 10 most frequently published authors in each of three ophthalmic subspecialty fields: glaucoma, macular, and corneal disease, for the years 2014 and 2015. Papers were selected with the single search 'topic' term Glaucoma, Macular, or Corneal, then manually filtered to exclude abstracts or book chapters and to include only articles written in English, French, German, Italian, and 
Dutch, in which at least one of us is fluent as it was important that we could ascertain the relevance of the financial interest of the author to the content of each article. As it is not our intention to single out, praise, nor criticize any specific author, we chose to select the authors at random and maintain their anonymity: by flipping a coin (i.e., we listed the ten most published authors in order of publication count and, starting with the most published author, we flipped a coin for each author and selected the first five heads), we randomly selected five of the ten most frequently published authors in each subspecialty. We excluded authors who did not report any conflicts of interests on any paper published in 2014 and $2015(\mathrm{~N}=2)$ and replaced them with the next author down the list, continuing to use the flip coin technique. To further safeguard the anonymity of the authors, we will not report the number of publications for each individual, as this may allow identification of several of the randomly selected authors by a simple search process, and our purpose is not to assign praise or blame to any individual author. For these fifteen authors identified at random, we pulled every peer reviewed publication published in 2014 and 2015, the year prior to and the year following the aforementioned seminal editorial. Two ophthalmologists independently reviewed each paper for relevance to the subspecialty subject matter and the discovered financial interests of the relevant author. These methods and filters yielded a total of 642 peer reviewed journal articles. We then analyzed each paper for availability to the reader of study funding or material support not specific to any author, and for internal consistency of author-specific DOI. Each author's COI were determined by review of previous literature in the same subspecialty area within the past year, thus falling within Bartlett and Liesegang's proposed three year reporting requirement. To establish the completeness of DOI, we first established author-specific COIs by looking at the DOIs present in included papers. 
graded the DOI of each paper included in our review as either unreported (i.e., COI relevant to the topic were declared by this author in a prior publication, but none were reported in the analyzed publication), incomplete (COI relevant to the topic were declared by this author in a prior publication, and some but not all such COI were reported in the analyzed publication), or complete (all COI previously declared by this author were declared in the analyzed publication).

Concurrent with exploring the consistency in authors' declarations of interests, we sought to understand the differences in the journals' treatment of DOI. We therefore analyzed the website of the 33 journals (i.e., all journals which published 3 or more of the randomly selected papers included in our study) to analyze their requirements of declaration of interests. We initiated the search manually by first searching the website of each journal to locate the guidelines for authors, or the 'editorial and publishing policies'. We searched 'Guidelines for authors' and 'editorial and publishing policies' for the keywords 'conflict', 'declar-', 'interest', 'fund', 'competing', 'monetary', 'financial', 'commercial', 'disclos-', and 'ICMJE' to ensure that we captured all relevant passages. Passages included for analysis comprised sections and external links or forms in which the search retrieved our keywords in a relevant manner (e.g., include "... may be influenced by a secondary interest." but exclude "... the subject must be of interest to a broad readership."). For each included journal, we captured whether the ICMJE Form for Disclosure of Potential Conflicts of Interest or an equivalent was required, whether a definition of conflicts of interests was provided, whether declared conflicts of interests were published alongside the publications and whether conflicts of interests may disbar a manuscript from publication. 


\section{Results:}

$\underline{\text { Authors: }}$

A random sampling of the most productive authors in ophthalmology yielded 642 peer reviewed articles which satisfied our inclusion criteria. In $64 \%$ of the articles, known conflicts of interest were unreported as determined by comparing internal consistency by author (Fig $\mathbf{1}$ ). In a further $25 \%$, the DOI was incomplete, and only $11 \%$ of articles reported a comprehensive list of industry affiliations according to the criteria advocated by the editors of the leading journals in the field.

Conversely, non-author specific general funding sources, such as grants or university support, were commented on in $78.7 \%$ of articles, leaving the reader uninformed in only $21.3 \%$ of the articles reviewed. The conflicts of interest declaration rates varied slightly by subspecialty, with glaucoma themed publications not reporting known COI in $54 \%$ of articles, corneal disease in $77 \%$, and macular disease in $60.6 \%$.

Journals:

To have sufficient representability, we reviewed DOI requirements and policies for journals that published three or more of the randomly selected articles included in our study; 524 articles were thus included in the journal analyses. In the 524 articles included in the journal analysis, approximately a third $(\mathrm{N}=186)$ were published in journals either requiring or endorsing the ICMJE form for DOI or an equivalent slightly modified DOI form. For this subset of papers, the declaration of known conflicts of interest were complete, incomplete, and unreported in $18 \%$, 30\%, and 53\% respectively (Fig 2). Of the remaining 338 papers published in journals in which a formal DOI form was not mandatory, the declaration of known conflicts of interest were complete, incomplete, and unreported in $12 \%, 27 \%$, and $61 \%$. 
The joint editorial by Bartley and Liesegang in November 2014 seems to have had no significant effect as the rate of denying known COI was $63 \%$ for articles published in 2014 and $61 \%$ for those published in 2015 (Fig 3).

Of the 33 journals investigated, only seven were listed on the ICMJE website as 'journals following ICMJE recommendations' (two of which were members through the association of journals they are part of rather than at the individual journal level). Interestingly, only two of the journals listed on the ICMJE website required that authors fill in the ICMJE Form for Disclosure of Potential Conflicts of Interest. Nonetheless, six journals not mentioned on the ICMJE website did require the ICMJE Form for Disclosure of Potential Conflicts of Interest before publication.

Almost a third $(\mathrm{N}=10)$ of the included journals required that authors fill in the ICMJE Form for Disclosure of Potential Conflicts of Interest or an equivalent form. About two third of the journals did not require all authors to fill in a DOI form, half of which did not mention the ICMJE in any of their policies. Figure 4 shows the different requirements of the included journals regarding ICMJE requirements and policies.

\section{Discussion:}

In medical sciences, conflicts of interest have the potential to undermine our genuine understanding of medical mechanisms, impede research, and ultimately harm patients. ${ }^{8}$ The medical literature continues to be disturbed by withdrawals of articles following revelations of financial impropriety, ghostwriting, and even data fabrication. ${ }^{9}$ The atmosphere of distrust between scientists and the general public continues to grow and the collegiality between academic medical researchers continues to erode. This has often led to salacious attacks in the lay press and, unavoidably, drawn attention from politicians. Unsurprisingly, authors with COI 
are 10 to 20 times more likely not to report negative findings in comparison to authors without COI. ${ }^{10}$ If the scientific community does not improve its self-policing efforts, outside enforcement may become inevitable. As Liesegang and Bartley suggest, improved transparency through declarations of interests by every author, whether they are deemed relevant or not, is one step towards improved self-regulation., ${ }^{1,2}$

Between the choices of the authors and those of the editors, the ophthalmologist consumer of scientific information is misinformed with regards to DOI in an astonishing $89 \%$ of peer reviewed journal articles. Our results provide empirical support to the concerns about consistency in declarations of COI raised by the editors of Ophthalmology and The American Journal of Ophthalmology. Differences between journals in their instructions for authors and requirements for declaring interests may help explain some of the COI reporting inconsistencies. Our view is that, as much as authors have a duty to declare all COI and comply with journals' policies, journals have a responsibility to endorse complete and coherent COI guidelines and to enforce them. Specifically, editors should pay closer attention to COI declarations of authors and put in place serious consequences in cases of misrepresentation and inconsistency. In turn, editors and journals who inadequately monitor COI — either by failing to notice inconsistencies, by endorsing loose regulations, or by incompletely publishing declared interests (i.e., in deciding which disclosures are relevant and which are not worth publishing) — may need to be held accountable by higher scientific communities such as national boards for research ethics (e.g., the US Office of Research Integrity, UK Research Integrity Office, etc.) or international organizations such as the ICMJE, the Committee on Publication Ethics (COPE), or the European Commission. ${ }^{11}$ 
Academic freedom and researcher's impartial quest for knowledge are essential to academic research. Nonetheless, because financial support from commercial for-profit entities is vital to research, demanding independence from such income sources would render many if not most research difficult to fund, at least in the current environment. Therefore, in order to preserve the authenticity of the knowledge captured by researchers, transparency, rather than independence, is put forward as the summum bonum. ${ }^{12,13}$ By requiring researchers to declare their financial, commercial, and all other potentially conflicting interests to the scientific community as their audience, scientific journals hope to give readers the necessary information to allow them to accurately estimate the credibility of the reported work and to judge the work in a more informed manner. $^{6}$

Transparency is not the end-all be-all of ethical scientific reporting. Rather, it serves as a starting point for asking questions and to improve the atmosphere of trust essential to the fiduciary relationship between the researchers and the clinician consumers of their data. One practical step suggested by our data would be a banding together of all ophthalmic, optometric, and vision science journals to standardize and optimize DOI requirements, a process which must be made more formal and with an emphasis both on facilitating proper DOI and enforcing consequences of improper disclosures according to the criteria proposed by Bartley and Liesegang.

\section{Abbreviations and Acronyms:}

COI: Conflict of interest

DOI: declaration of interest

ICMJE: International Committee of Medical Journal Editors 


\section{Financial Support}

JS has received a travel grant from the UatB Center for Clinical Ethics Patrick Romanell fund to present this data at ASBH 2016.

\section{References:}

1. Liesegang, Thomas J., and George B. Bartley. (2014). Footnotes, Acknowledgments, and Authorship: Toward Greater Responsibility, Accountability, and Transparency.

Ophthalmology 121, no. 12: 2297-298. doi:10.1016/j.ophtha.2014.09.010.

2. Liesegang, Thomas J., and George B. Bartley. (2014). Toward Transparency of Financial Disclosure. Ophthalmology 121, no.: 2077-078. doi:10.1016/j.ophtha.2014.08.039.

3. Blum, Jared A. (2009). Requirements and Definitions in Conflict of Interest Policies of Medical Journals. JAMA 302, no. 20: 2230. doi:10.1001/jama.2009.1669.

4. Ancker, Jessica S., and Annette Flanagin. (2007). A comparison of conflict of interest policies at peer-reviewed journals in different scientific disciplines. Science and Engineering Ethics 13, no. 2: 147-57. doi:10.1007/s11948-007-9011-z.

5. "ICMJE Form for Disclosure of Potential Conflicts of Interest."

http://www.bing.com/cr?IG=68D9D7BB7732418EB9C6BFDA7C74CD48\&CID=17F23 FF1C6896D91105F3585C7196CDB\&rd=1\&h=LcytXzGpa1aARq8t68vcCQPa-xR7-KmwaHaxhh3NjA\&v=1\&r=http\%3a\%2f\%2fwww.nejm.org\%2fdoi\%2fsuppl\%2f10.1056 \%2fNEJMc1413069\%2fsuppl_file\%2fnejmc1413069_disclosures.pdf\&p=DevEx,5069.1. 
6. Baethge, Christopher. (2013). The effect of a conflict of interest disclosure form using closed questions on the number of positive conflicts of interest declared - a controlled study. PeerJ 1. doi:10.7717/peerj.128.

7. McCoy, Matthew S., and Ezekiel J. Emanuel. (2017). Why There Are No 'Potential' Conflicts of Interest. JAMA 317, no. 17: 1721-22. doi:10.1001/jama.2017.2308

8. Bion, Julian. (2009). Financial and intellectual conflicts of interest: confusion and clarity. Current Opinion in Critical Care 15, no. 6: 583-90. doi:10.1097/mcc.0b013e328332f53a.

9. Patel, Alpesh A., Peter G. Whang, Andrew P. White, Michael G. Fehlings, and Alexander R. Vaccaro. (2011). Pitfalls in the publication of scientific literature: a road map to manage conflict of interest and other ethical challenges. Journal of Neurosurgery 114, no. 1: 21-26. doi:10.3171/2010.8.jns091834.

10. Friedman, Lee S., and Elihu D. Richter. (2004). Relationship between conflicts of interest and research results. Journal of General Internal Medicine 19, no. 1: 51-56. doi:10.1111/j.1525-1497.2004.30617.x.

11. Probst, Pascal, Felix J.Hüttner, Ulla Klaiber, Markus K. Diener, Markus W. Büchler, and Phillip Knebel. (2015). Thirty years of disclosure of conflict of interest in surgery journals. Surgery 157, no. 4: 627-33. doi:10.1016/j.surg.2014.11.012.

12. Cosgrove, Lisa, Sheldon Krimsky, Emily E. Wheeler, Shannon M. Peters, Madeline Brodt, and Allen F. Shaughnessy. (2016). Conflict of Interest Policies and Industry Relationships of Guideline Development Group Members: A Cross-Sectional Study of Clinical Practice Guidelines for Depression. Accountability in Research 24, no. 2: 99115. doi:10.1080/08989621.2016.1251319. 
13. Krimsky, Sheldon. (2013). Do Financial Conflicts of Interest Bias Research? Science, Technology, \& Human Values 38, no. 4: 566-87. doi:10.1177/0162243912456271. 
Figure 1 Total Declarations of Interest (DOI\}

DOI $(\mathrm{n}=642)$

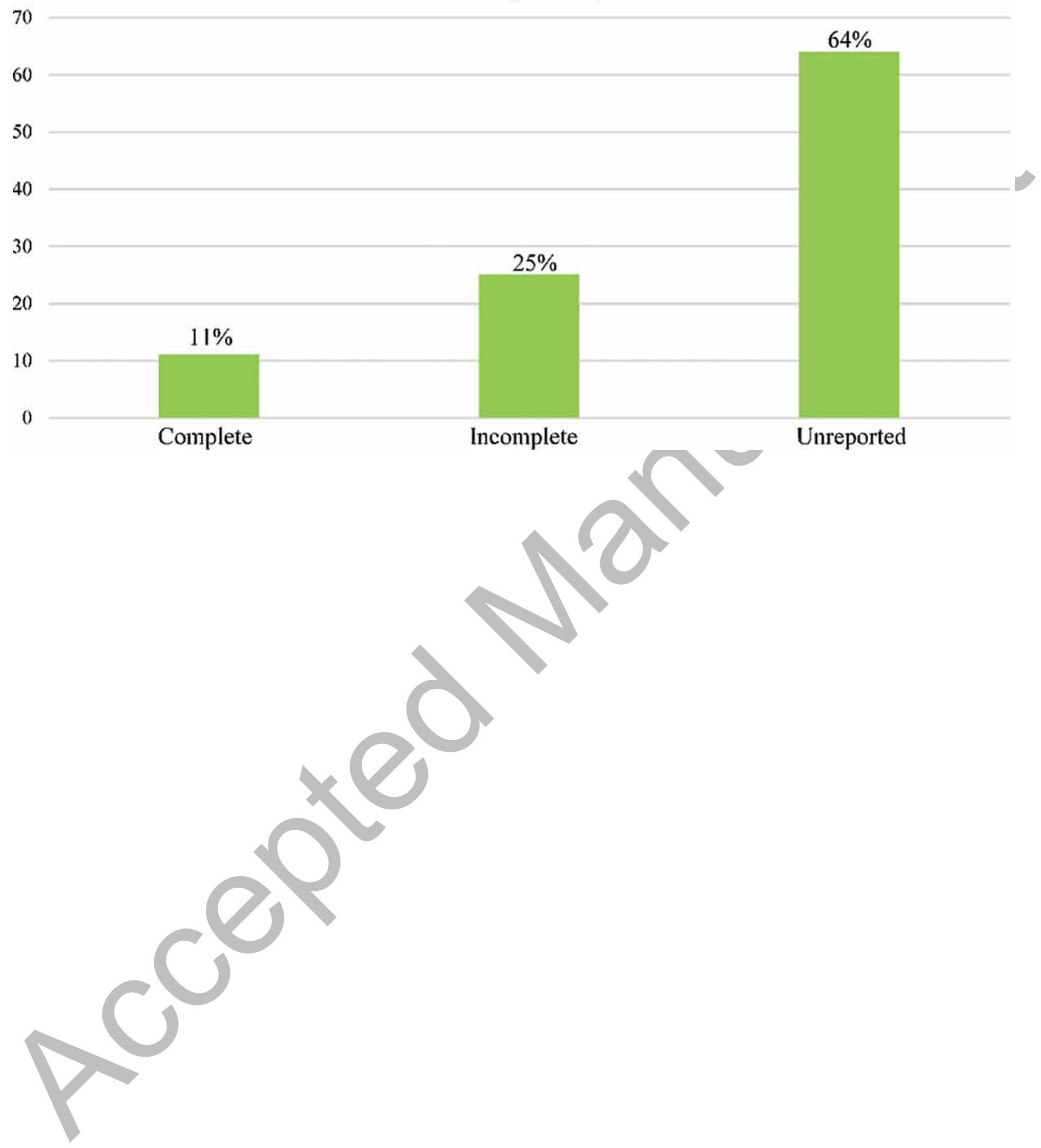


Figure 2. Percentage of Complete, Incomplete, and Unreported $\mathrm{CO} /$ in Journals with Different DOI Requirements
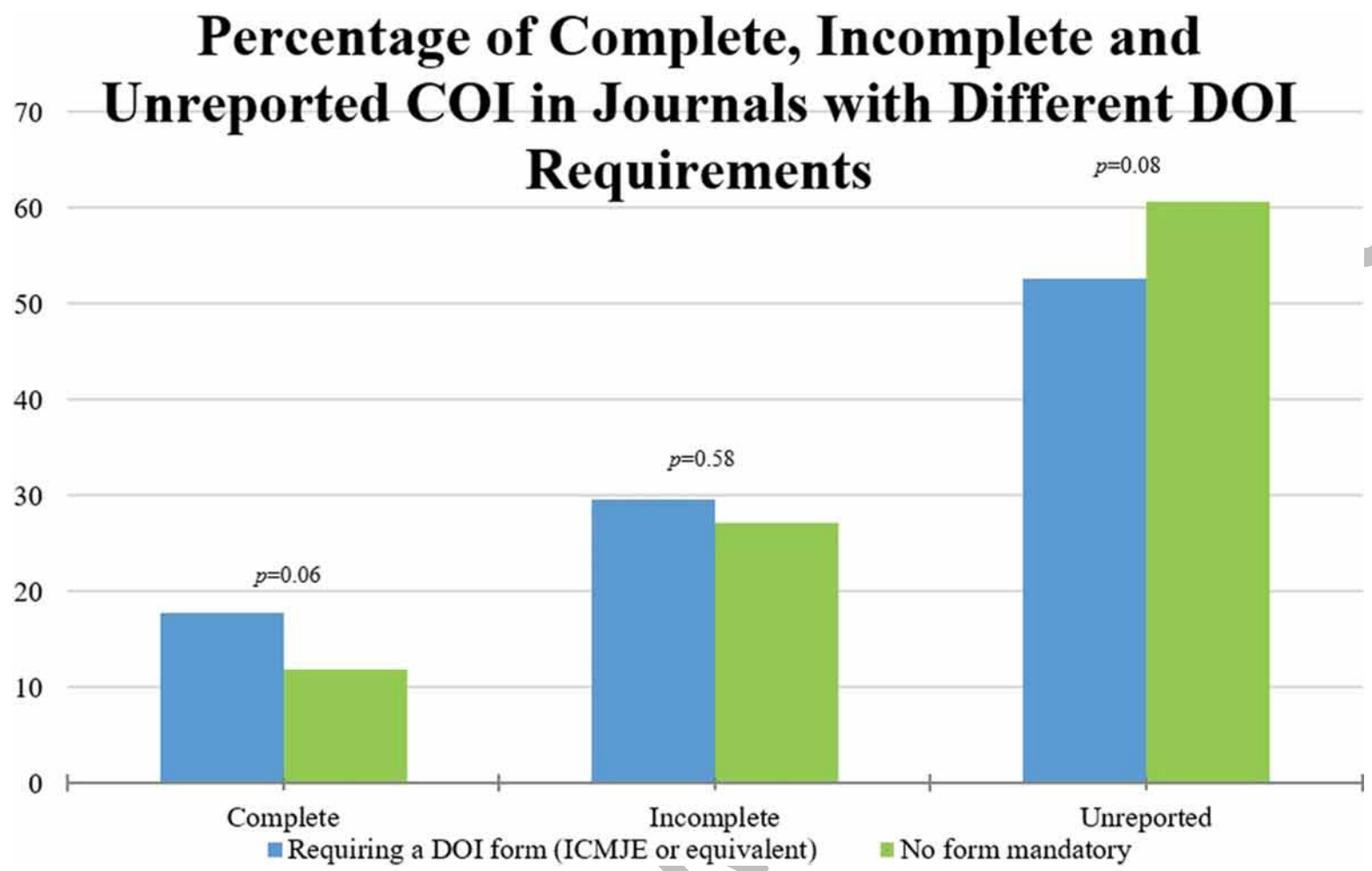
Figure 3 Percentage of Complete, Incomplete, and Unreported COi: 2014 vs 2015 Percentage of Complete, Incomplete, and Unreported COI: 2014 vs 2015

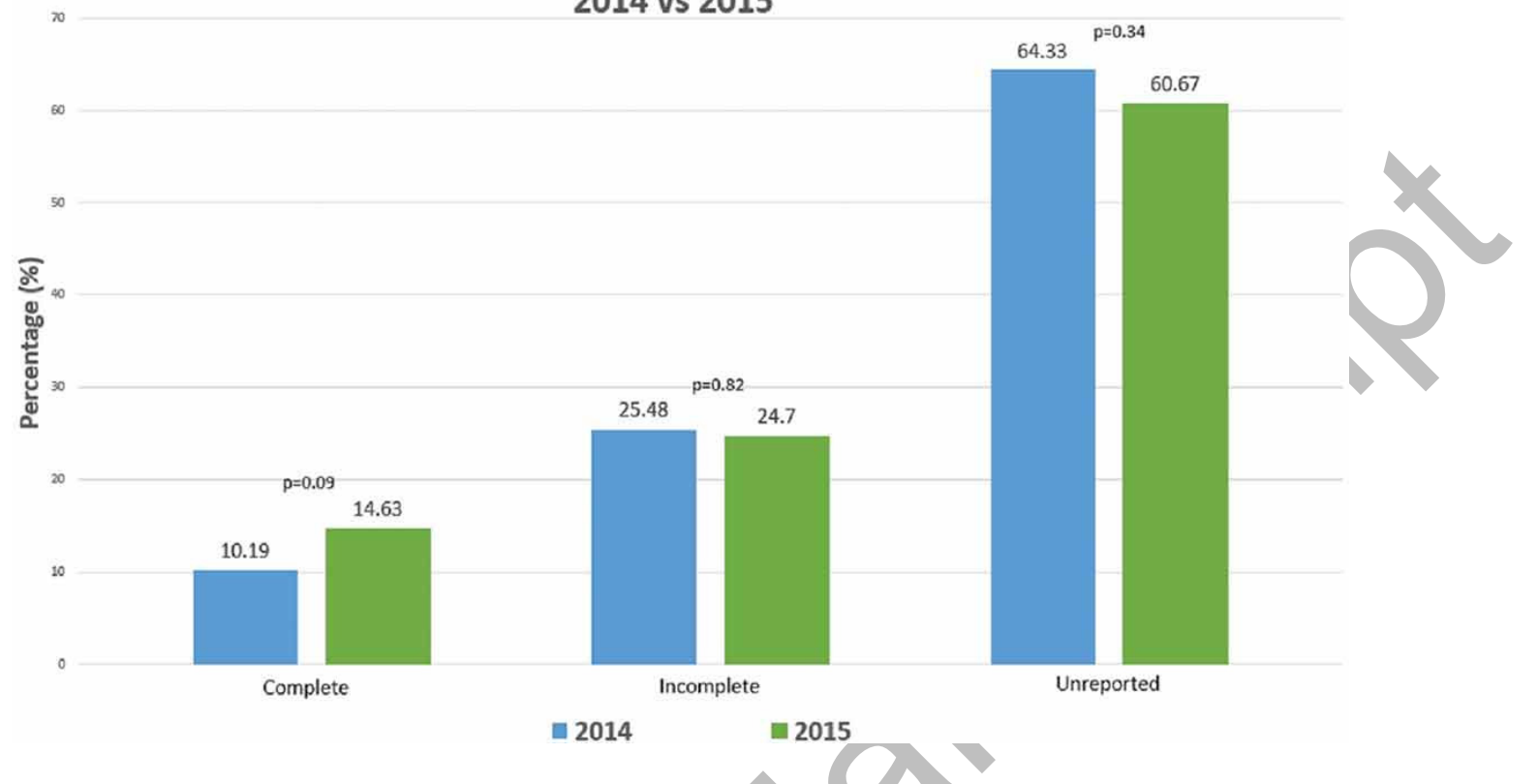


Figure 4 ICMJE Form Requirement

\section{ICMJE form requirement}

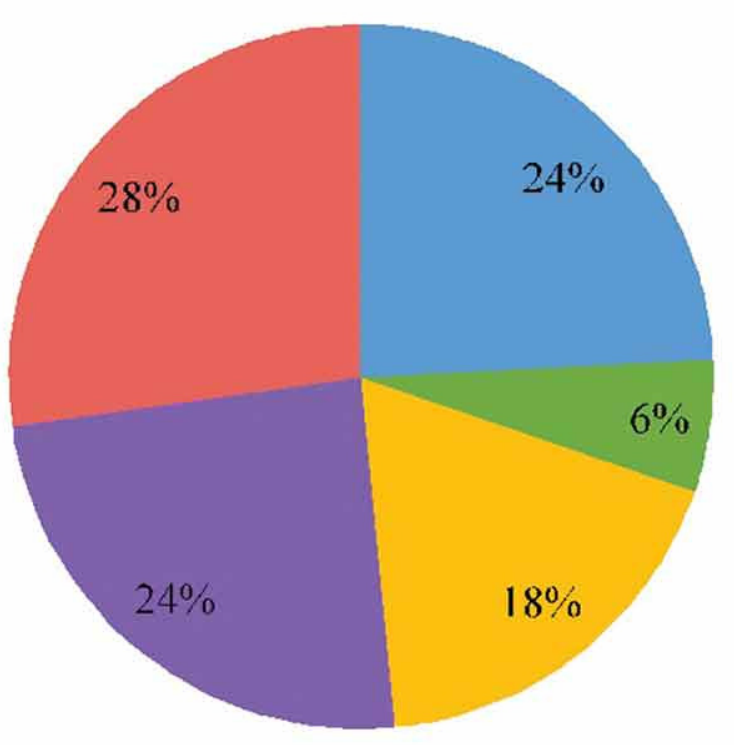

- ICMJE form mandatory

n ICMJE-like form mandatory

- ICMJE form/policy recommended

- ICMJE mentioned but not in $\mathrm{COI}$

a No mention 